\title{
Aportes a la determinación de la Huella ambiental de biocombustibles en Argentina. Influencia de los sistemas de manejo de cultivos sobre el impacto del consumo de agua, del uso del suelo y de las emisiones de gases de efecto invernadero para el biodiesel de soja
}

\author{
R. Piastrellini1 ${ }^{1, *}$ \\ (1) CLIOPE, Universidad Tecnológica Nacional, Facultad Regional Mendoza - Consejo Nacional de Investigaciones Científicas y Tecnológicas CONICET. \\ Cnel. Rodríguez 273 (5500), Ciudad de Mendoza, Argentina \\ *Autor de correspondencia: R. Piastrellini [roxana.ppp@gmail.com]
}

> Recibido el 27 de abril de 2016 - Aceptado el 11 de julio de 2016

Piastrellini, R. 2016. Aportes a la determinación de la Huella ambiental de biocombustibles en Argentina. Influencia de los sistemas de manejo de cultivos sobre el impacto del consumo de agua, del uso del suelo y de las emisiones de gases de efecto invernadero para el biodiesel de soja. Ecosistemas 25(2): 106-109. Doi.: 10.7818/ECOS.2016.25-2.14.

\section{Introducción}

El Análisis de Ciclo de Vida (ACV) es una de las metodologías más difundidas para evaluar los efectos ambientales derivados de la producción de biocombustibles. La Huella ambiental (HA) es un indicador de ciclo de vida que abarca diversos impactos ambientales relacionados con las emisiones al ambiente, y el uso, agotamiento y degradación de recursos escasos. La metodología del ACV y la HA han sido gestadas en países desarrollados, atendiendo a las problemáticas ambientales, las condiciones tecnológicas, las regulaciones y las prioridades allí imperantes. Su aplicación en regiones con características diferentes a las de estos países presenta ciertas limitaciones, ya que los modelos no cuentan con procedimientos sencillos para incorporar las particularidades locales. Un ejemplo de ello es la inclusión de las prácticas agrícolas propias del sistema productivo con mayor extensión en la Argentina: la siembra directa. Omitir estas particularidades podría conducir a obtener resultados desfavorables desde el punto de vista ambiental, y consecuentemente competitivo, para los agrocombustibles argentinos.

De lo expuesto surge la siguiente Hipótesis de trabajo: "La aplicación estándar de la metodología de ACV para la evaluación ambiental de biocombustibles no evidencia las ventajas que presenta la producción del biodiésel de soja (Glycine max) argentino". El objetivo de este estudio es contribuir en la búsqueda de soluciones metodológicas para la correcta consideración de las características regionales productivas, durante la evaluación del ciclo de vida del biodiésel de soja en la Argentina. Específicamente, se analiza la posibilidad de incorporar las prácticas agrícolas regionales en las metodologías que permiten estimar los flujos de inventario y evaluar los impactos ambientales, haciendo especial hincapié en las emi- siones de gases de efecto invernadero (GEI), y los impactos potenciales de uso del suelo y de uso consuntivo de agua.

Análisis de Ciclo de Vida de los biocombustibles para el transporte: estado del arte

Son diversas las prácticas agrícolas que pueden modificar los flujos de GEI del suelo a la atmósfera (Piastrellini et al. 2014). Sin embargo, la mayoría de los estudios sobre ACV de biocombustibles se limitan a contabilizar las tasas de fertilizaciones. El enfoque del IPCC (2006) es el más aceptado para estimar las emisiones de GEl en estos estudios. En Argentina, el Instituto Nacional de Tecnología Agropecuaria ha adoptado este enfoque para evaluar la producción de soja (y sus derivados) bajo distintos sistemas de labranza. No obstante, otros autores afirman que los valores de emisiones que arroja el método sobrestiman las mediciones a campo (Álvarez et al. 2014), realzando la necesidad de validar metodologías alternativas.

Gran parte de las publicaciones sobre impactos de uso del suelo evalúan la contribución de la expansión del cultivo energético al calentamiento global. Pocos autores utilizan metodologías específicas para estudios de ciclo de vida de productos, entre ellos Milà i Canals et al. (2013). Sin embargo, no contemplan la influencia de las técnicas de labranza y manejo del cultivo.

Por otro lado, vasta cantidad de publicaciones confirman la influencia de los sistemas de labranza en la disponibilidad de agua en el suelo. A pesar de ello, se registra sólo un estudio de ACV que analiza los efectos de las labranzas en el consumo de agua (Chukalla et al. 2015). Este estudio se focaliza en la evaporación del suelo, sin considerar otros parámetros relevantes como la escorrentía superficial. 


\section{Metodología}

EI ACV se realiza con un enfoque "de la cuna a la puerta" (Fig. 1). Las emisiones de GEI se evalúan con la metodología del IPCC (2006). Además, se analiza un modelo alternativo para estimar los flujos de $\mathrm{N}_{2} \mathrm{O}$, desarrollado por el Joint Research Centre (JRC) (Koeble y Leip 2010). En la evaluación de impactos de uso del suelo se aplican las recomendaciones de la guía UNEP-SETAC (Koellner et al. 2013), diseñada específicamente para estudios de ciclo de vida. Se evalúan los servicios ecosistémicos que resultan afectados por modificaciones en los flujos de $C$ entre el suelo y la atmósfera (Piastrellini et al. 2015). El uso consuntivo de agua se estima en base al modelo de Hoekstra et al. 2011. Se realizan ajustes en la evapotranspiración, la escorrentía superficial y la productividad del cultivo. Para ello, se aplican los enfoques de Allen et al. (1998) y Littleboy et al. (1999).

\section{Resultados}

\section{Emisiones de GEI}

El modelo del JRC arroja resultados de emisiones de $\mathrm{N}_{2} \mathrm{O}$ que cuadruplican a los obtenidos con el modelo del IPCC y, por consiguiente, a las emisiones medidas a campo en la región sojera argentina (Tabla 1). Este incremento en las emisiones se traduce en mayores contribuciones a diferentes impactos ambientales, especialmente al calentamiento global.

Los resultados de emisiones totales de GEI muestran que el biodiesel de soja argentino es ventajoso con respecto al diésel fósil que sustituye, con porcentajes de reducción de $75.4 \%-80.5 \%$, según el sistema agrícola considerado. Estos valores duplican a los publicados por organismos internacionales para el biodiesel de soja argentino (Fig. 2).

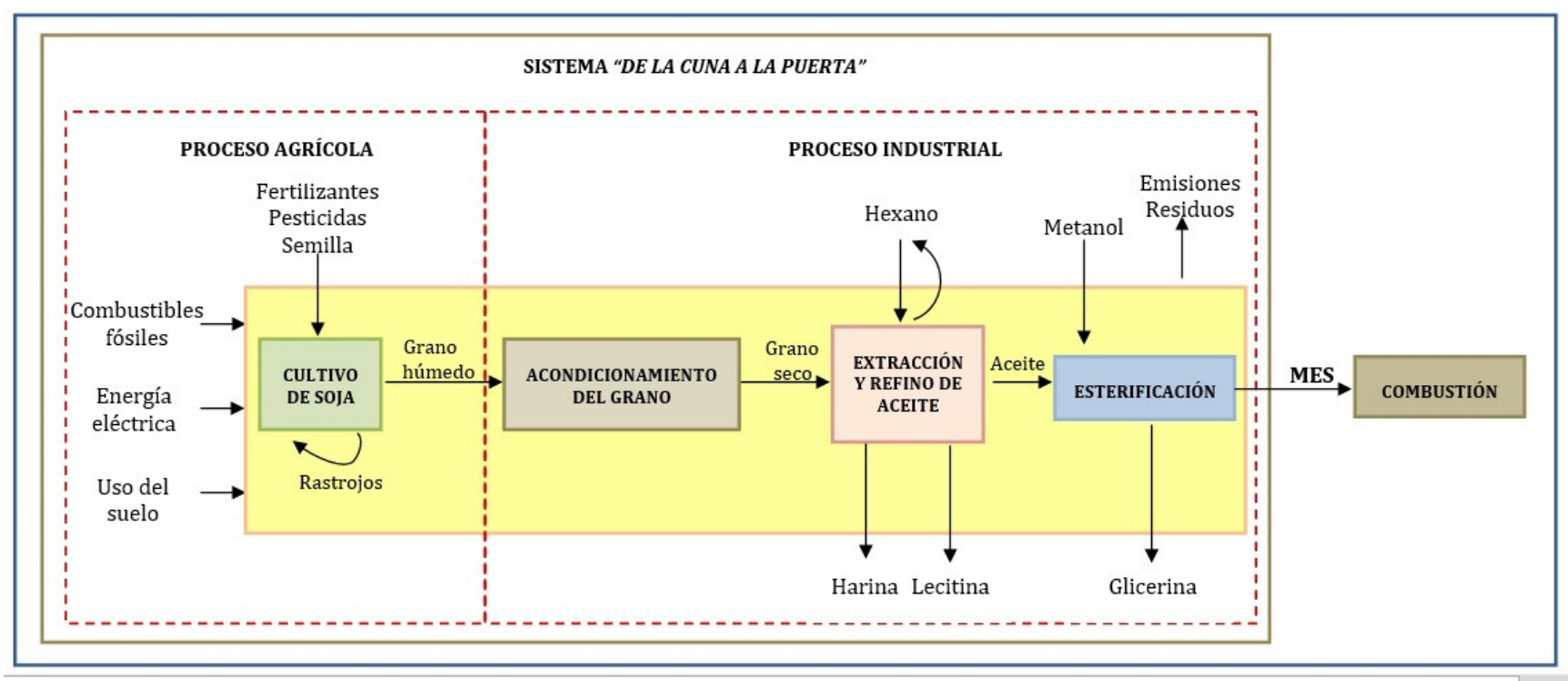

Figura 1. Diagrama de flujo simplificado del ciclo de vida del biodiesel de soja (metil éster de soja-MES), teniendo en cuenta las tecnologías más difundidas en Argentina. El diagrama representa un enfoque "de la cuna a la puerta", el cual considera las etapas comprendidas entre la extracción de los insumos necesarios para la fabricación del producto hasta la salida de la planta de fabricación.

Figure 1. Process flow diagram of the life cycle of soybean biodiesel (soybean methyl ester-MES) considering the most widespread technologies in Argentina. The diagram represents an cradle-to-gate approach, ie from resource extraction (cradle) to the factory gate.

Tabla 1. Emisiones de $\mathrm{N}_{2} \mathrm{O}$ de suelos cultivados con soja en Argentina (en $\mathrm{kg} \mathrm{N} \mathrm{N}_{2} \mathrm{O} / \mathrm{t}$ grano de soja), para distintos sistemas agrícolas: $S D$-SP: soja de primera en siembra directa, en condiciones de secano; SD-SS: soja de segunda en siembra directa, en condiciones de secano; $S D-S P$-RS: soja de primera en siembra directa bajo riego suplementario, con manejo integrado de plagas; LC-SP: soja de primera en labranza convencional, en condiciones de secano.

Table 1. $\mathrm{N}_{2} \mathrm{O}$ emissions from soils cultivated with soybean in Argentina (in $\mathrm{kg} \mathrm{N} \mathrm{N}_{2} \mathrm{O} / \mathrm{t}$ soybeans), for different agricultural systems: SD-SP: early soybean in no-tillage under rainfed conditions; SD-SS: late soybean in no-tillage under rainfed conditions; SD-SP-RS: early soybean in no-tillage under supplementary irrigation with integrated pest management; LC-SP: early soybean in conventional tillage under rainfed conditions.

Emisiones de $\mathrm{N}_{2} \mathrm{O}$ estimadas en base al IPCC (kg/t grano)

Sistema agrícola

\begin{tabular}{cccccc}
\hline Directas & Indirectas & Totales & Directas & Indirectas & Totales \\
\hline 2.53 & 0.06 & 0.31 & 1.11 & 0.06 & 1.17 \\
0.26 & 0.06 & 0.32 & 1.41 & 0.06 & 1.47 \\
0.28 & 0.07 & 0.35 & 0.86 & 0.06 & 0.92 \\
0.26 & 0.06 & 0.31 & 1.30 & 0.06 & 1.36
\end{tabular}




\section{Impactos de uso del suelo}

Al considerar las particularidades de las tecnologías regionales de producción, los valores del impacto de ocupación y transformación del suelo sobre los servicios ecosistémicos se reducen entre un $15 \%$ y un $49 \%$, con respecto al escenario que no incorpora estos aspectos (Tabla 2).

Los resultados muestran que el sistema productivo adoptado en la región sojera argentina permite mantener la capacidad de la tierra para producir y sostener biomasa útil y la capacidad de los ecosistemas para captar carbono del aire en mejores niveles que las tecnologías de producción implementadas en otras regiones del mundo.

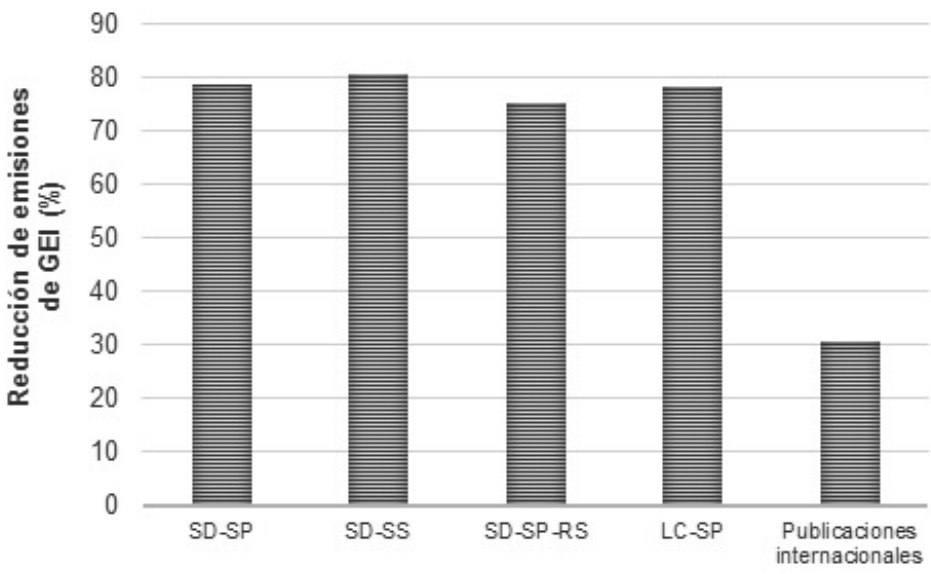

Figura 2. Reducción de emisiones de GEl del biodiesel de soja argentino con respecto al diésel fósil. El proceso agrícola corresponde a: SD-SP: soja de primera en siembra directa, en condiciones de secano; SD-SS: soja de segunda en siembra directa, en condiciones de secano; SD-SP-RS: soja de primera en siembra directa bajo riego suplementario, con manejo integrado de plagas; LC-SP: soja de primera en labranza convencional, en condiciones de secano.

Figure 2. Reduction in GHG emissions of Argentine soybean biodiesel, relative to fossil diesel. The agricultural process corresponds to: SD-SP: early soybean in no-tillage under rainfed conditions; SD-SS: late soybean in notillage under rainfed conditions; SD-SP-RS: early soybean in no-tillage under supplementary irrigation with integrated pest management; LC-SP: early soybean in conventional tillage under rainfed conditions.

\section{Impactos del uso consuntivo del agua}

Los resultados confirman que las técnicas de manejo agrícola propias de la región sojera argentina presentan ventajas frente a las técnicas agrícolas convencionales: disminuyen las pérdidas por evapotranspiración, mejora la capacidad de retención hídrica del suelo, se prolonga el período de almacenamiento de agua en el suelo y se retarda el proceso de escurrimiento (Fig. 3). Esto genera mayor cantidad de agua disponible para satisfacer los requerimientos hídricos, la cual es aprovechada por el cultivo aumentando el consumo de agua, y consecuentemente la productividad. Al relacionar el consumo de agua con la productividad del cultivo, resulta que el impacto ambiental es menor para el sistema que incorpora las características regionales que para el sistema que no las considera (Fig. 4).

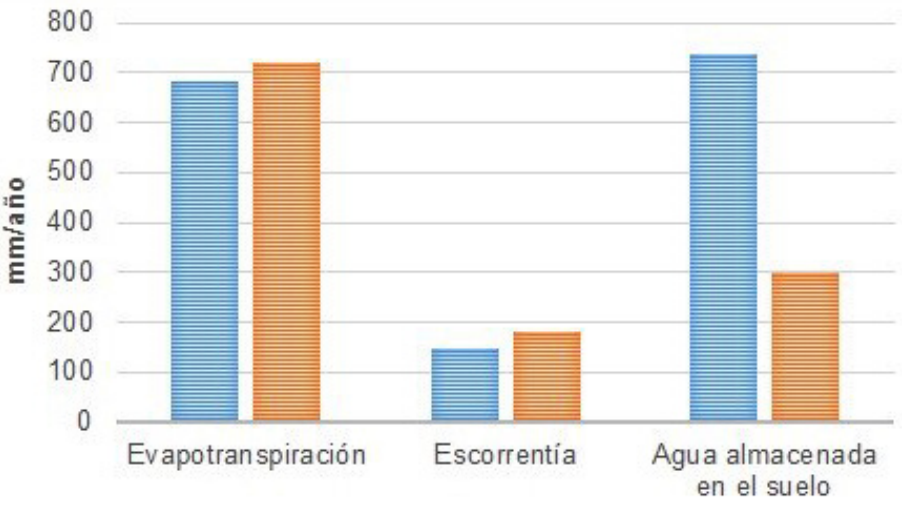

EEscenario con ajustes 톨 Escenario sin ajustes

Figura 3. Evapotranspiración del cultivo, escorrentía superficial y agua almacenada en el suelo correspondiente a un ciclo de producción de soja en Argentina. El escenario con ajustes incluye modificaciones metodológicas asociadas a la tecnología soja de primera en siembra directa, con $80 \%$ de cobertura de rastrojos. El escenario sin ajustes corresponde al sistema de labranza convencional, con ausencia de rastrojos en superficie.

Figure 3. Crop evapotranspiration, surface runoff and soil water stored corresponding to one soybean production cycle in Argentina. Scenario with adjustments (in blue) includes methodological changes related to early soybean in no-tillage, with $80 \%$ of stubble coverage. Scenario without adjustments (in orange) corresponds to early soybean in conventional tillage, without crop residues on the surface.

Tabla 2. Impactos de ocupación y transformación del suelo del biodiesel de soja argentino sobre el Potencial de producción biótica (PPB) y el Potencial de regulación del clima (PRC). El PPB se refiere a la capacidad de la tierra para producir y sostener biomasa útil, mientras que el PRC se refiere a la capacidad de los ecosistemas para captar carbono del aire.

Table 2. Impacts of land occupation and land transformation of Argentine soybean biodiesel on Biotic production potential (PPB) and Climate regulation potential (PRC). The PPB refers to the capacity of the land to produce and sustain useful biomass, while the PRC refers to the capacity of ecosystems to uptake carbon from air.

Ajustes metodológicos

Soja de primera en siembra directa

Secuencia de doble cultivo trigo/soja

Sin ajustes metodológicos
0.09

0.05

0.10
23.10

*Los impactos de transformación cuantifican los efectos de posibles cambios en el uso del suelo por la expansión de la zona sojera hacia la Selva de las Yungas.

** Ce: carbono equivalente de combustión fósil. 


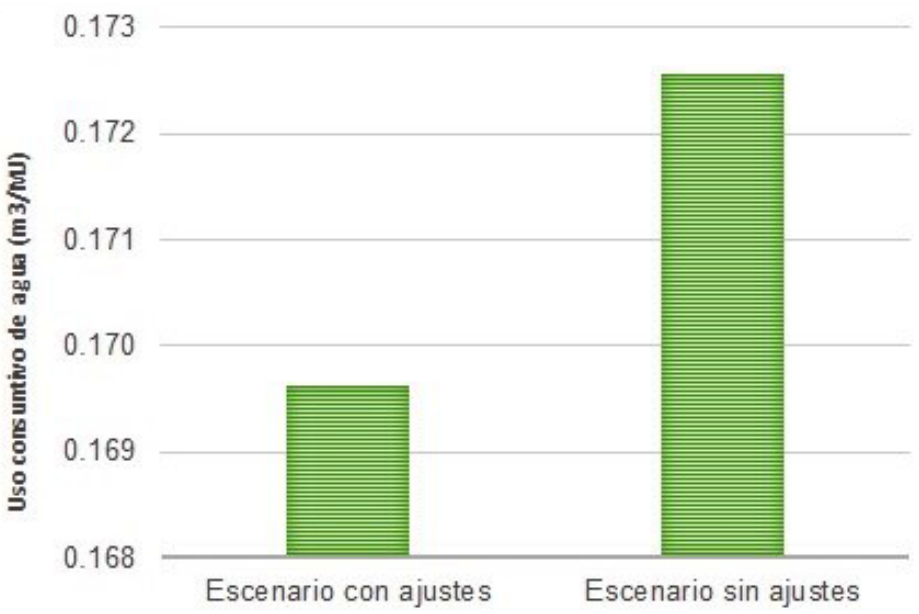

Figura 4. Uso consuntivo de agua de la producción de biodiesel de soja en Argentina. El escenario con ajustes incluye modificaciones metodológicas asociadas a la tecnología soja de primera en siembra directa, con $80 \%$ de cobertura de rastrojos. El escenario sin ajustes corresponde al sistema de labranza convencional, con ausencia de rastrojos en superficie.

Figure 4. Consumptive water use of soybean biodiesel production in Argentina. "Escenario con ajustes" includes methodological changes related to early soybean in no-tillage, with $80 \%$ of stubble coverage. "Escenario sin ajustes" corresponds to early soybean in conventional tillage, without crop residues on the surface.

\section{Conclusiones}

Este trabajo demuestra que las técnicas de labranza y de manejo del cultivo adoptadas en Argentina brindan al biodiesel de soja importantes beneficios ambientales; y que la no inclusión de las características distintivas de estas prácticas durante la estimación de los flujos de emisiones y la evaluación de los potenciales impactos de ciclo de vida, representa serias deficiencias en los procedimientos de cálculo ya que modifican notablemente los resultados.

Los ajustes metodológicos propuestos en ésta tesis son aplicables no solo al caso de estudio sino también a otros biocombustibles y productos de base agrícola que implementen técnicas de producción similares.

\section{Referencias}

Allen, R.G., Pereira, L.S., Raes, D., Smith, M. 1998. Crop evapotranspiration: guidelines for computing crop water requirements. Irrigation and Drainage Paper n. 56. FAO, Roma, Italia.

Álvarez, C., Cosentino, V., Costantini, A., Álvarez, C.R., Taboada, M.A. 2014. Efecto de la secuencia de cultivo y la siembra directa sobre las emisiones de óxido nitroso. En: Pascale Medina, C., Zubillaga, M., Taboada, M.A. (eds), Suelos, producción agropecuaria y cambio climático: avances en la Argentina. Ministerio de Agricultura, Ganadería y Pesca de la Nación, Buenos Aires, Argentina.

Chukalla, A.D., Krol, M.S., Hoekstra, A.Y. 2015. Green and blue water footprint reduction in irrigated agriculture: effect of irrigation techniques, irrigation strategies and mulching. Hydrology and earth system sciences 19(12), 4877.

Hoekstra, A.Y., Chapagain, A.K., Aldaya, M.M., Mekonnen, MM. 2011. The water footprint assessment manual: setting the global standard. Earthscan, Londres, Reino Unido - Washington, Estados Unidos.

IPCC-Intergovernmental Panel on Climate Change 2006. Guidelines for $\mathrm{Na}-$ tional Greenhouse Gas Inventories. Prepared by the National Greenhouse Gas Inventories Programme, IGES, Japón.

Koeble, R., Leip, A. 2010. Global pattern of $\mathrm{N}_{2} \mathrm{O}$ emissions from soils due to cultivation of potential biofuel crops. Joint Research Centre, Comisión Europea. Disponible en: ftp://mars.jrc.ec.europa.eu/Afoludata/Public/ DS221/N2O subjrc global.v02 20100519.pdf.

Koellner, T., de Baan, L., Beck, T., Brandão, M., Civit, B., Goedkoop, M., et al 2013. Principles for life cycle inventories of land use on a global scale. The International Journal of Life Cycle Assessment 18 (6): 1203-1215.

Littleboy, M., Freebairn, D.M., Woodruff, D.R., Silburn, D.M., Hammer, G.L. 1999. PERFECT Version 3.0. A computer simulation model of Productivity, Erosion, Runoff Functions to Evaluate Conservation Techniques. Boletín QB89005, Department of Primary Industries, Brisbane, QLD, Australia.

Milà i Canals, L.M., Rigarlsford, G., Sim, S. 2013. Land use impact assessment of margarine. International Journal of Life Cycle Assessment 18 1265-1277.

Piastrellini, R., Arena, A.P., Civit, B. 2014. Análisis de Ciclo de Vida de la cadena productiva de la soja (Glycine max) como cultivo energético. En: Pascale Medina, C., Zubillaga, M., Taboada, M.A. (eds), Suelos, producción agropecuaria y cambio climático: avances en la Argentina, pp. 515-525. Ministerio de Agricultura, Ganadería y Pesca de la Nación, Buenos Aires, Argentina.

Piastrellini, R., Civit, B., Arena, A.P. 2015. Influence of agricultural practices on Biotic production potential and Climate regulation potential. A case study for Life cycle assessment of soybean (Glycine max) in Argentina. Sustainability 7, 4386-4410.

\section{ROXANA PIASTRELLINI}

Aportes a la determinación de la Huella ambiental de biocombustibles en Argentina. Influencia de los sistemas de manejo de cultivos sobre el impacto del consumo de agua, del uso del suelo y de las emisiones de gases de efecto invernadero para el biodiesel de soja.

Tesis Doctoral

Universidad Tecnológica Nacional - Facultad Regional Mendoza, Argentina

Mayo 2015

Directores: Alejandro Pablo Arena

Publicaciones resultantes de la tesis

Civit, B., Arena, AP., Piastrellini, R., Curadelli, S., Silva Colomer, J. 2011. Comparación entre la huella hídrica de biodiesel obtenido a partir de aceite de colza y aceite de soja. Avances en Energías Renovables y Medio Ambiente 15:127-134

Piastrellini, R., Arena, A.P., Civit, B. 2014. Análisis de Ciclo de Vida de la cadena productiva de la soja (Glycine max) como cultivo energético. En: Pascale Medina, C., Zubillaga, M., Taboada, M.A. (eds), Suelos, producción agropecuaria y cambio climático: avances en la Argentina, pp. 515-525. Ministerio de Agricultura, Ganadería y Pesca de la Nación, Buenos Aires, Argentina.
Piastrellini, R., Civit, B., Arena, A.P. 2015. Influence of agricultural practices on Biotic production potential and Climate regulation potential. A case study for Life cycle assessment of soybean (Glycine max) in Argentina. Sustainability 7, 4386-4410.

Piastrellini, R., Civit B., Arena, A.P. 2016. Soybean (Glycine max) production, in no tillage, $A R(\mathrm{~kg})$. LCADB.SUDOE (Southwest of Europe) Database. Disponible en: http://lcadb.sudoe.ecotech.cat/. 